\title{
REVISTAMATĒRIA
}

ISSN 1517-7076 artigo e11798, 2017

\section{Comportamento reológico do Bio-PE e do PCL na presença do PEgAA e PEgMA}

\author{
Rheological behaviour of Bio-PE and PCL \\ upon addition of PEgAA and PEgMA
}

\author{
Elieber Barros Bezerra ${ }^{1}$, Danyelle Campos de França ${ }^{2}$,Dayanne Diniz de Souza Morais ${ }^{3}$, \\ Eduardo Silva Barbosa Ferreira ${ }^{4}$, Edcleide Maria Araújo ${ }^{5}$, \\ Renate Maria Ramos Wellen ${ }^{6}$
}

\author{
${ }^{1-5}$ Universidade Federal de Campina Grande - UFCG, CEP: 58429-900, Campina Grande, PB. \\ e-mail: elieberbarros@hotmail.com;danyellecampos_1@hotmail.com; dayannediniz@hotmail.com; eduar- \\ dosbf95@gmail.com; edcleide.araujo@ufcg.edu.br \\ ${ }^{6}$ Universidade Federal da Paraíba- UFPB,CEP: 58297-000, João Pessoa , PB. \\ e-mail: wellen.renate@gmail.com
}

\section{RESUMO}

O reômetro de torque é um equipamento que reproduz em escala laboratorial o processamento de polímeros. Neste equipamento, a velocidade de rotação dos rotores, temperatura e tempo de processamento são parâmetros predeterminados e impostos ao sistema investigado; avalia-se o torque necessário para a fusão, mistura e homogeneização da amostra. Através do gráfico do torque em função do tempo e do gráfico da temperatura em função do tempo de processamento, cujos valores estão relacionados com as propriedades reológicas, estrutura e massa molar das amostras, é possível obter informações sobre o comportamento dos polímeros em fluxo, ou seja, durante o processamento. Neste trabalho, foi investigado o efeito do polietileno graftizado com ácido acrílico (PEgAA) e do polietileno graftizado com anidrido maléico (PEgMA) no biopolietileno (Bio-PE) e no poli( $\varepsilon$-caprolactona) (PCL) por reometria de torque. Foi verificado que o PEgAA e o PEgMA promoveram aumento no torque dos sistemas Bio-PE/PEgAA, Bio-PE/PEgMA, PCL/PEgAA e PCL/PEgMA, possivelmente resultante de interações e/ou reações químicas ocorridas nesses sistemas poliméricos. Espectros de FTIR evidenciaram possíveis interações e/ou reações químicas entre os grupos funcionais dos polímeros grafitizados e o Bio-PE e o PCL. Valores de torque mais altos foram observados nos sistemas com parâmetros de solubilidade $(\delta)$ mais próximos, corroborando com os cálculos propostos por Hansen.

Palavras-chave: Bio-PE, PCL, PEgAA, PEgMA, reometria de torque, parâmetro de solubilidade.

\begin{abstract}
The torque rheometer is equipment that enables to simulate the polymer processing in a laboratory scale. A set of parameters, i.e., rotor speed, temperature and processing time are predefined and imposed to the compounds under analysis. The necessary torque for melting and mixing the compound is measured. Through the plots of torque as a function of time and temperature, which are linked to the rheological properties, microstructure and molar weight of the samples, it is possible to acquire information regarding the flow behavior of polymers, meaning during processing. In this work, the effect of acrylic acid-grafted polyethylene (PEgAA) and maleic anhydride-grafted polyethylene (PEgMA) in bio-polyethylene (Bio-PE) and in poly( $\varepsilon$ caprolactone) (PCL) was performed by torque rheometry. PEgAA and PEgMA increased the torque rheometry values of the polymeric systems under investigation, i.e, Bio-PE/PEgAA, Bio-PE/PEgMA, PCL/PEgAA and PCL/PEgMA, possibly due to chemical interaction and/or reactions occurred during the processing. FTIR spectra presented possible interactions and/or chemical reactions between the functional groups of grafted polymers and Bio-PE and PCL. Higher torques were observed for the systems with similar solubility parameters agreeing with the mathematical calculations provided by Hansen.
\end{abstract}

Keywords: Bio-PE, PCL, PEgAA, PEgMA, torque rheometry, solubility parameter.

\section{INTRODUÇÃO}


A indústria de polímeros constitui hoje um dos pilares do estilo de vida contemporâneo. É enorme a quantidade de bens produzidos de material polimérico, por serem eles utilizados em quase todas as áreas das atividades humanas, nas indústrias automobilísticas (pneus e para-choques), de embalagens (vasilhames e envoltos para alimentos), de revestimentos, de vestuário, médico-hospitalar (próteses, dispositivos para liberação de drogas), para citar alguns exemplos. Isto é consequência também do fato de que os materiais poliméricos vêm conquistando muitos mercados através da substituição de produtos metálicos e cerâmicos por poliméricos, por estes apresentarem maior praticidade a um custo inferior [1-3]. Dentre os diferentes tipos de polímeros, o biopolietileno (Bio-PE) é um biopolímero proveniente de uma fonte renovável, a cana-de-açúcar. O Bio-PE apresenta as mesmas características químicas, mecânicas e de processabilidade do polietileno de origem petroquímica, sendo assim ambos possuem a mesma versatilidade de aplicações e são $100 \%$ recicláveis [4-6].

Um grupo de polímeros que vem despertando interesse científico e tecnológico é o de polímeros biodegradáveis, estes são materiais que podem ser degradados no meio ambiente por ação de microrganismos em um período de tempo curto (semanas ou meses sob condições favoráveis) $[7,8]$. O policaprolactona (PCL) é um polímero biodegradável com aplicações tecnológicas (próteses ortopédicas, embalagens de alimentos, dispositivos para liberação controlada de fármaco, etc). O PCL tem como características tenacidade, flexibilidade e boa compatibilidade com vários polímeros (poli(ácido lático) (PLA), polihidroxibutirato (PHB), etc), sendo utilizado de forma frequente como aditivo e carga para melhorar características de processamento e propriedades finais (como por exemplo, a resistência ao impacto) [9]. A produção deste polímero tem um custo elevado em relação aos polímeros de uso corrente, como o PE e o polipropileno (PP). Entretanto, espera-se que com o aumento da utilização do PCL e novas rotas de processamento, seu custo diminua tornando-o mais competitivo financeiramente $[7,8,10,11]$.

No tangente ao desenvolvimento de novas formulações, a produção de blendas poliméricas vem sendo amplamente utilizada como um método economicamente viável para a modificação de materiais poliméricos já existentes. A mistura de dois ou mais polímeros e/ou copolímeros formando uma blenda pode resultar numa combinação ótima das propriedades intrínsecas de cada componente, gerando compostos com características não encontradas num único polímero $[12,13]$. As blendas poliméricas podem ser miscíveis, imiscíveis, e/ou compatíveis. Uma das características de misturas imiscíveis poliméricas é a formação de distintas fases, o que pode conduzir a baixas propriedades mecânicas. Sistemas imiscíveis podem ser compatibilizados através de agentes interfaciais: um copolímero em bloco, graftizado ou, outro polímero que contenha grupos reativos com ambos os componentes do sistema imiscível. A função do agente interfacial é promover estados de mistura onde as propriedades finais da blenda estejam de acordo com os valores desejados, ou seja, reduzindo a tensão interfacial, permitindo um menor tamanho da fase dispersa, estabilizando a morfologia contra efeitos térmicos ou de cisalhamento durante as etapas de processamento e fornecendo adesão no estado sólido. Muitas vezes o sucesso atingido no desenvolvimento de blendas poliméricas é acompanhado de vantagens econômicas para o mercado de materiais poliméricos [12-15].

Segundo o banco de dados da literatura, a reometria de torque pode fornecer evidências da ocorrência ou não de reações químicas entre os componentes da blenda e o compatibilizante. Na adição do compatibilizante à blenda imiscível, os seguintes resultados podem ser registrados por reometria de torque: a) ocorrência de reação entre pelo menos um dos componentes da blenda e o grupo funcional do compatibilizante; isto é indicado pelo aumento do torque devido ao aumento da viscosidade do sistema, promovido por ligações químicas intramoleculares e/ou extensão da cadeia formando um copolímero in situ; b) ausência de reação entre os componentes da blenda e o grupo funcional do compatibilizante; normalmente, sem aparente alteração do torque; c) ocorrência de degradação em pelo menos um dos componentes da blenda ou do copolímero formado in-situ; conduzindo a diminuições no valor do torque $[9,16,17]$.

Diante desse contexto, o objetivo deste trabalho foi investigar a eficiência da adição de dois copolímeros funcionalizados: o polietileno enxertado com ácido acrílico (PEgAA) e o polietileno enxertado com anidrido maléico (PEgMA) no comportamento reológico de um biopolímero e um polímero biodegradável, biopolietileno (Bio-PE) e o policaprolactona (PCL), respectivamente.

\section{MATERIAIS E MÉTODOS}

\subsection{Materiais Utilizados}

Os materiais utilizados neste trabalho foram:

- Polietileno de Alta Densidade (Bio-PE), I'm green® SHC7260, Braskem. Polímero produzido a partir da cana-de-açúcar. Conteúdo mínimo de carbono proveniente de fonte renovável de 
94\%. Densidade de $0,959 \mathrm{~g} / \mathrm{cm}^{3}, \mathrm{IF}=7,2 \mathrm{~g} / 10 \min \left(190^{\circ} \mathrm{C} / 2,16 \mathrm{~kg}\right)$.

- Poli ( $\varepsilon$-caprolactona) (PCL), Capa ${ }^{\circledR} 6500, \mathrm{IF}=28 \mathrm{~g} / 10 \mathrm{~min}\left(190^{\circ} \mathrm{C} / 2,16 \mathrm{~kg}\right)$ e alongamento até $800 \%$, fabricada pela Perstorp Winning Fórmulas.

- Polietileno enxertado com 1,5 - 1,7\% de Anidrido Maléico (PEgMA) Polybond 3029, Addivant. Densidade de $0,95 \mathrm{~g} / \mathrm{cm}^{3}, \mathrm{IF}=4,0 \mathrm{~g} / 10 \mathrm{~min}\left(190^{\circ} \mathrm{C} / 2,16 \mathrm{~kg}\right)$ e temperatura de fusão $\left(\mathrm{T}_{\mathrm{m}}\right)$ $=130^{\circ} \mathrm{C}$.

- Polietileno enxertado com 5,5 - 6,5\% de Ácido Acrílico (PEgAA) Polybond 1009, Addivant. Densidade de $0,95 \mathrm{~g} / \mathrm{cm}^{3}, \mathrm{IF}=5,0 \mathrm{~g} / 10 \mathrm{~min}\left(190^{\circ} \mathrm{C} / 2,16 \mathrm{~kg}\right)$ e $\mathrm{T}_{\mathrm{m}}=127^{\circ} \mathrm{C}$.

\subsection{Reometria de torque}

Os experimentos de reometria de torque foram realizados em um misturador interno Rheocord 600 acoplado a um equipamento HAAKE System 90, com rotores do tipo roller a uma velocidade de $60 \mathrm{rpm}$ e temperatura de $200^{\circ} \mathrm{C}$, sob atmosfera de ar durante 10 minutos. A massa total dentro da câmara de mistura foi de $50 \mathrm{~g}$.

As blendas Bio-PE/PEgAA, Bio-PE/PEgMA, PCL/PEgAA, PCL/PEgMA foram produzidas nas condições acima citadas, as seguintes composições foram estudadas: 90/10, 80/20, 70/30, 50/50 (\% em peso).

\subsection{Espectroscopia na Região do Infravermelho com Transformada de Fourier (FTIR)}

As análises de FTIR do Bio-PE, do PCL, dos polímeros enxertados e das blendas foram realizadas em um espectrômetro modelo Spectrum 400 FT Mid-IR da PerkimElmer, com varredura de 4000 a $400 \mathrm{~cm}^{-1}$, operando no modo ATR.

\section{RESULTADOS E DISCUSSÃO}

A reometria de torque foi a técnica empregada para avaliar a estabilidade térmica, reatividade e a processabilidade dos sistemas de blendas produzidos neste trabalho. $\mathrm{O}$ torque é diretamente proporcional à viscosidade, e então à massa molar do sistema polimérico. Aumentos nos valores de torque podem ser interpretados como existência de extensão de cadeia, ligações cruzadas, interações entre grupos químicos das espécies químicas presentes. Evidências de reações de degradação podem também ser observadas por curvas de reometria de torque. Nesse último caso, tem-se uma diminuição do torque com o tempo. Para que estes processos possam ser determinados com confiabilidade, o processamento deve ocorrer num plateau constante de temperatura.

A Figura 1 (a) apresenta as curvas de torque em função do tempo de processamento para o Bio-PE, PCL, PEgAA e PEgMA. A Figura1 (b) ilustra uma ampliação do intervalo de processamento, 9 - 10 minutos, a fim de ilustrar melhor o torque das resinas. Pode-se observar que no Bio-PE, o torque em função do tempo é superior ao do PCL, indicando que a viscosidade do Bio-PE é superior. Nota-se que após a completa fusão destes polímeros (depois de 2 minutos) o torque se mantém constante, o que indica estabilidade térmica dos polímeros sob estas condições de processamento, ou seja, indicativo de ausência de processos degradativos.

Em relação aos polímeros PEgAA e PEgMA, o PEgMA apresentou valor de torque ligeiramente maior, indicativo de elevada viscosidade, o que está de acordo com os resultados de IF desses materiais, IF do PEgMA é $4.0 \mathrm{~g} / 10 \mathrm{~min}$ e o IF do PEgAA é $5.0 \mathrm{~g} / 10 \mathrm{~min}$ (dados do fornecedor). 


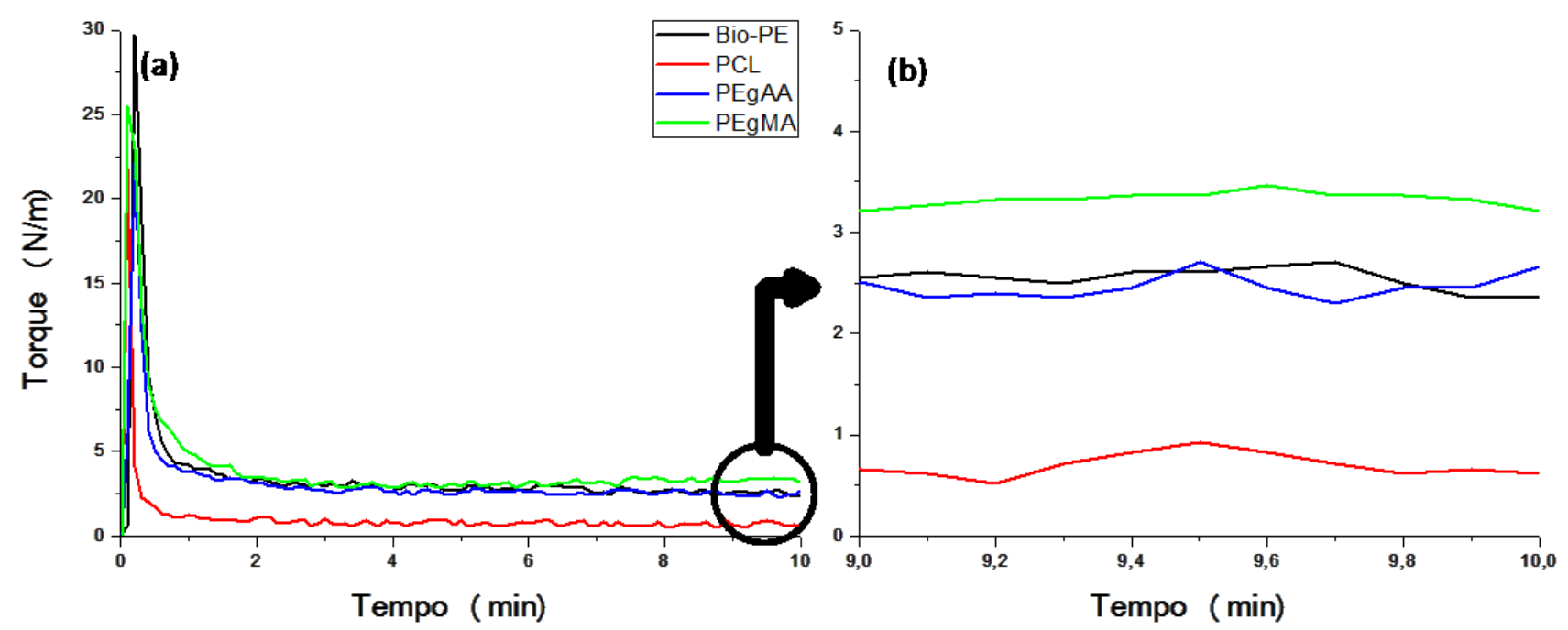

Figura 1: Curvas de torque em função do tempo de processamento dos polímeros e compatibilizantes utilizados neste trabalho.

De forma a garantir que as variações da viscosidade sejam resultantes do aumento ou redução da massa molecular, a temperatura de processamento deve ser mantida constante [17].

Na Figura 2, são ilustradas as curvas de temperatura versus tempo dos polímeros e compatibilizantes utilizados neste trabalho, para um tempo correspondente a 10 minutos de processamento. A partir destas curvas, observa-se uma queda inicial da temperatura ( 0 - 1 minuto) relacionada com a alimentação do material na câmara de mistura. Em seguida, houve o aumento da temperatura até atingir um plateau aproximadamente constante (após $5 \mathrm{~min}$ ), o que garante a confiabilidade dos resultados apresentados neste trabalho.

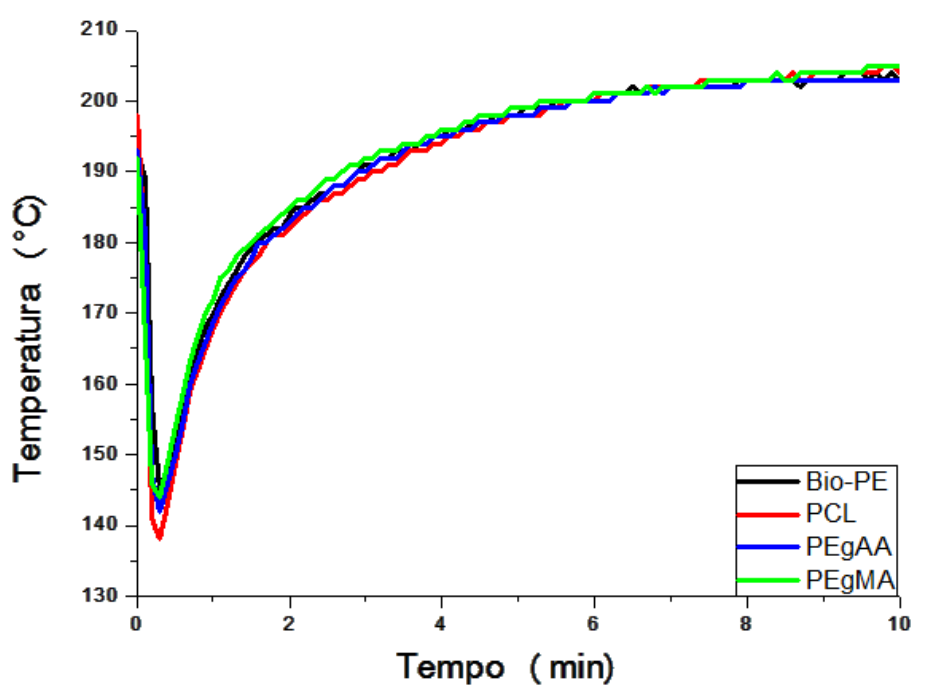

Figura 2: Curvas de temperatura em função do tempo de processamento dos polímeros e compatibilizantes utilizados neste trabalho.

Na Figura 3, pode-se observar o efeito da adição do PEgAA no comportamento reológico do Bio-PE. Nota-se que o aumento do teor de PEgAA promoveu um aumento na viscosidade desses sistemas. O aumento deste parâmetro pode estar relacionado aos seguintes mecanismos: um valor mais baixo do IF do PEgAA o que contribui para aumento da sua viscosidade, como também, à presença do grupo carboxílico no PEgAA que enrijece sua cadeia. Por isso, o aumento do teor de PEgAA (10 a 30\%) no Bio-PE tende a aumentar a 
viscosidade. Entretanto, a composição Bio-PE/PEgAA (50/50) apresentou viscosidade intermediária entre o

Bio-PE e os demais sistemas. É sabido que normalmente, os polímeros funcionalizados (por exemplo: PEgAA, PEgMA) têm baixa massa molar para também facilitar a sua difusão para a interface dos polímeros e, consequentemente, a compatibilização destes. Provavelmente, aumentando-se ainda mais o teor de PEgAA, a tendência será diminuir a viscosidade do sistema em função de sua baixa massa molar, até o valor do componente puro $[9,17,18]$.
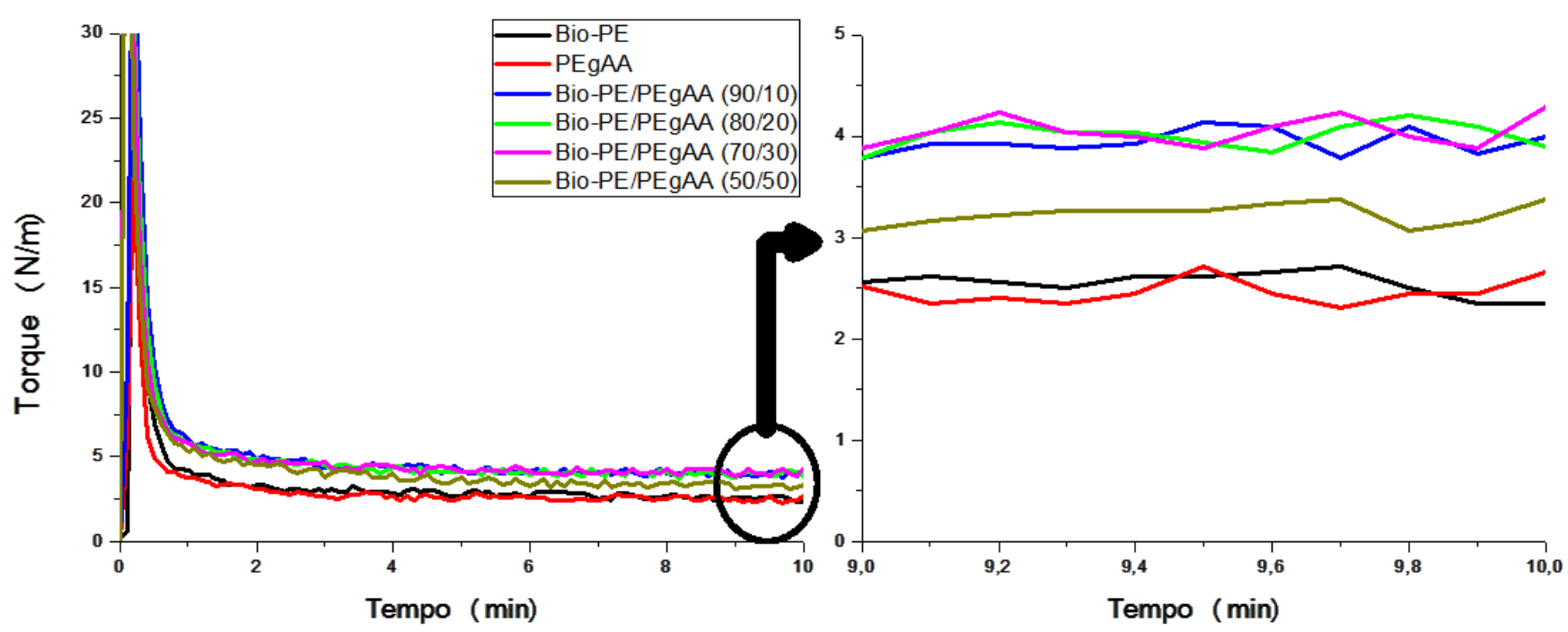

Figura 3: Curvas de torque em função do tempo de processamento do Bio-PE, e dos sistemas Bio-PE/PEgAA: 90/10,80/20, 70/30 e 50/50 (medidas em peso).

A Figura 4 ilustra o efeito da adição do PEgMA no comportamento de torque do Bio-PE. Verifica-se que os sistemas apresentam maior torque que o Bio-PE e o PEgMA puros, para todas as composições estudadas. Isto pode ser um indicativo de maior solubilidade entre os grupamentos MA e o Bio-PE, como também um valor mais baixo do IF do PEgMA o que contribui para aumento da viscosidade [12, 17, 18].
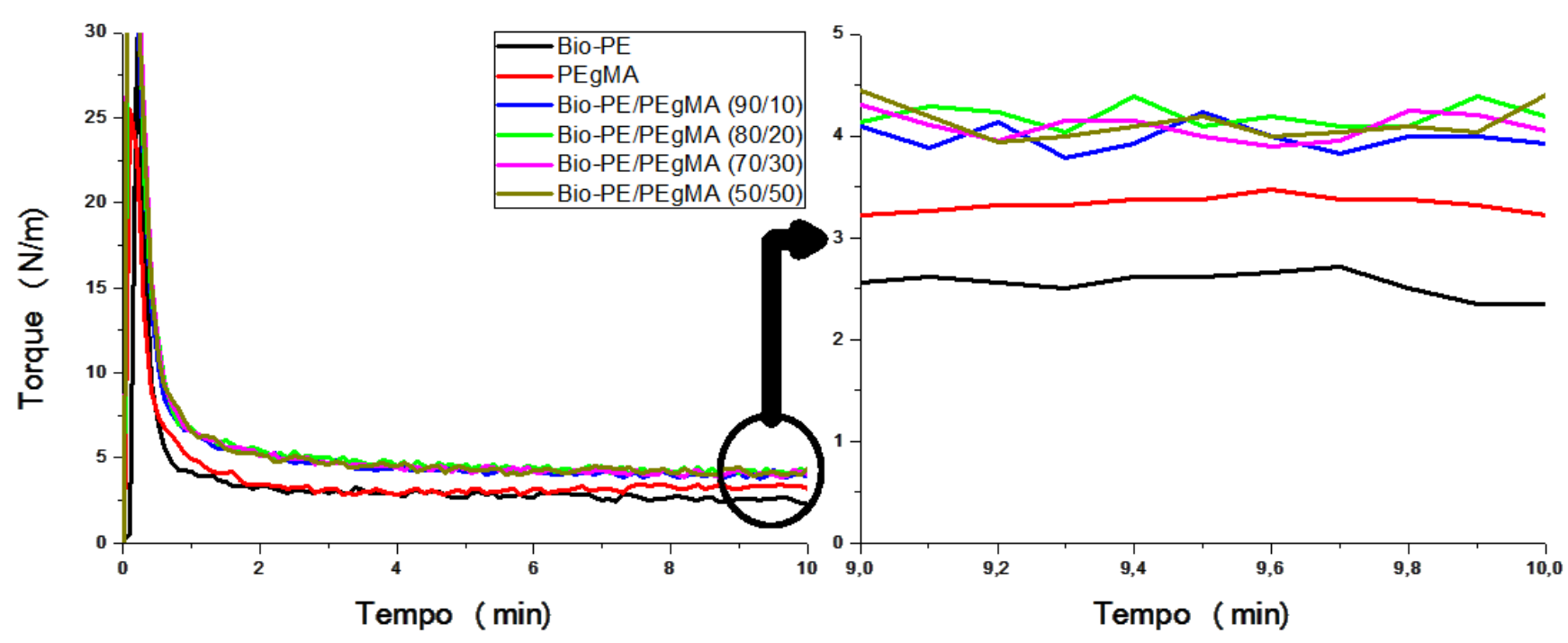

Figura 4: Curvas de torque em função do tempo de processamento do Bio-PE, e dos sistemas Bio-PE/PEgMA: 90/10,80/20, 70/30 e 50/50 (medidas em peso).

Na Figura 5 são ilustradas as curvas de temperatura versus tempo para o Bio-PE com os teores de 10, 
20, 30 e 50\% de PEgAA e PEgMA, respectivamente. Como já mencionado na discussão da Figura 2, observa-se inicialmente uma diminuição da temperatura relacionada com a alimentação do material na câmara de mistura, em seguida a temperatura aumenta e atinge um plateau constante garantindo desta forma a confiabilidade e reprodutibilidade dos resultados apresentados nesse trabalho.
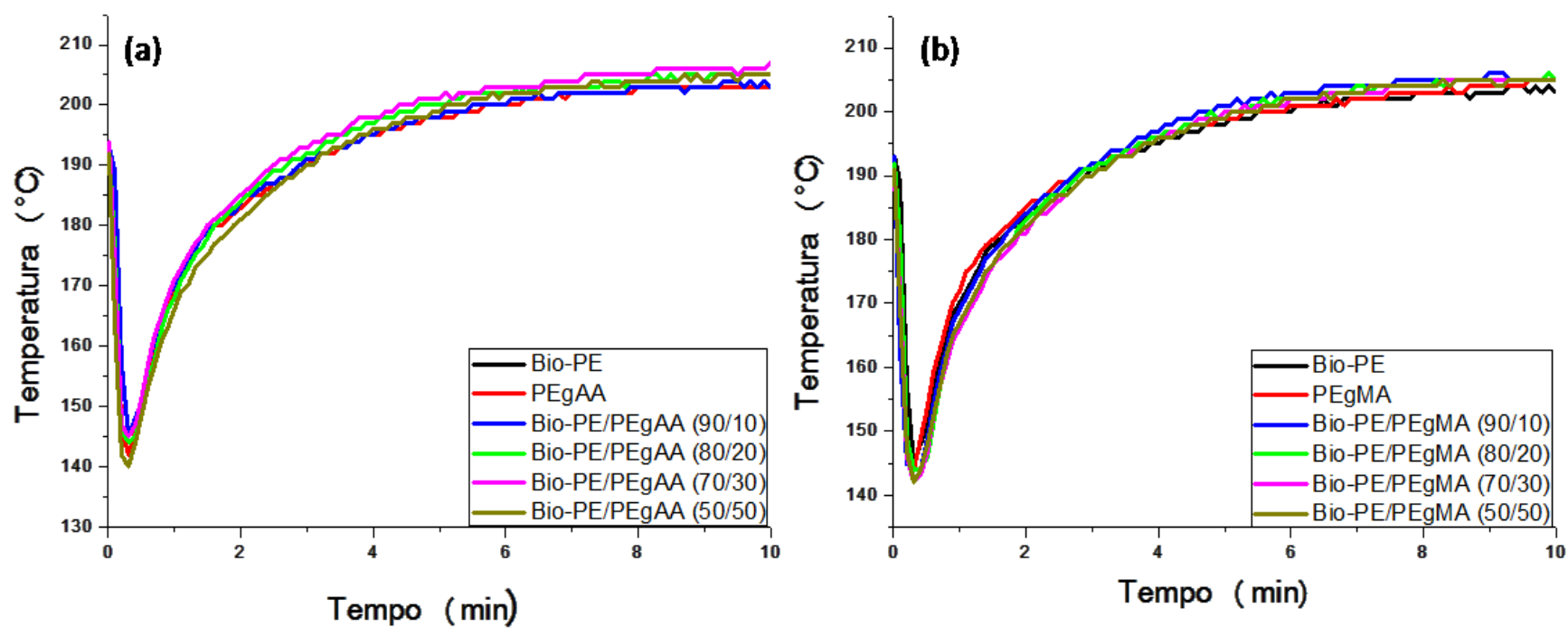

Figura 5: Curvas de temperatura em função do tempo de processamento: (a) Bio-PE/PEgAA e (b) Bio-PE/PEgMA.

Buscando indicativos da solubilidade dos sistemas poliméricos estudados nesse trabalho, cálculos do parâmetro de solubilidade do PEgAA e do PEgMA foram realizados de acordo com as Equações 1.1 e 1.2, respectivamente:

$$
\begin{aligned}
& \delta_{P E g A A}=w_{P E} \delta_{P E}+w_{A A} \delta_{A A} \\
& \delta_{P E g M A}=w_{P E} \delta_{P E}+w_{M A} \delta_{M A}
\end{aligned}
$$

Onde: $w_{P E}, w_{A A}, w_{M A}$ correspondem às frações volumétricas do $\mathrm{PE}$, do ácido acrílico (AA) e do anidrido maléico (MA), respectivamente. De acordo com os fabricantes $w_{A A}=5.5-6.5 \%$ e $w_{M A}=1.5-$ $1.7 \%$. $\delta_{P E}, \delta_{A A}$ e $\delta_{M A}$ são os parâmetros de solubilidade do PE, AA e do MA, neste trabalho foram utilizados os seguintes valores: $\delta_{P E}=15.76 \mathrm{MPa}^{0,5}, \delta_{A A}=24.6 \mathrm{MPa}^{0,5}$ e $\delta_{M A}=27.8 \mathrm{MPa}^{0,5}[19]$.

Os seguintes valores de parâmetro de solubilidade foram obtidos:

$$
\begin{aligned}
& \delta_{P E g A A}=16.29 M \mathrm{~Pa}^{0,5} \\
& \delta_{P E g M A}=15.96 M P a^{0,5}
\end{aligned}
$$

Quanto mais próximos forem os parâmetros de solubilidade maior a tendência de interações entre as espécies reagentes. Neste trabalho foi considerado que $\delta_{B i o-P E}=15.76 \mathrm{MPa}^{0,5}$. Desta forma, espera-se que o sistema Bio-PE/PEgMA interaja mais do que o Bio-PE/PEgAA [20]. Esta consideração está de acordo com as curvas de torque mostradas nas Figuras 3 e 4, e com os espectros de FTIR da Figura 11.

As Figuras 6 e 7 ilustram as curvas de torque em função do tempo do PCL e dos sistemas PCL/PEgAA e PCL/PEgMA. Os sistemas compatibilizados apresentaram viscosidade inferior aos compatibilizantes. Observa-se também que a adição do PEgAA e do PEgMA contribuiu para o aumento da viscosidade do PCL. De forma geral, o sistema PCL/PEgMA apresentou viscosidade um pouco superior em relação ao sistema PCL/PEgAA, provavelmente resultante de um valor mais baixo do IF do PEgMA o que contribui para aumento da viscosidade, bem como, maior interação molecular e reações químicas entre os agrupamentos do anidrido maléico e do PCL [16, 21-24].

O PCL possui $\delta_{P C L}=15.79 \mathrm{MPa}^{0,5}$ em temperaturas próximas ao do processamento das blendas, valor este mais próximo ao do PEgMA, $\delta_{P E g M A}=15.96 \mathrm{MPa}^{0,5}$, o que provavelmente contribui para uma 


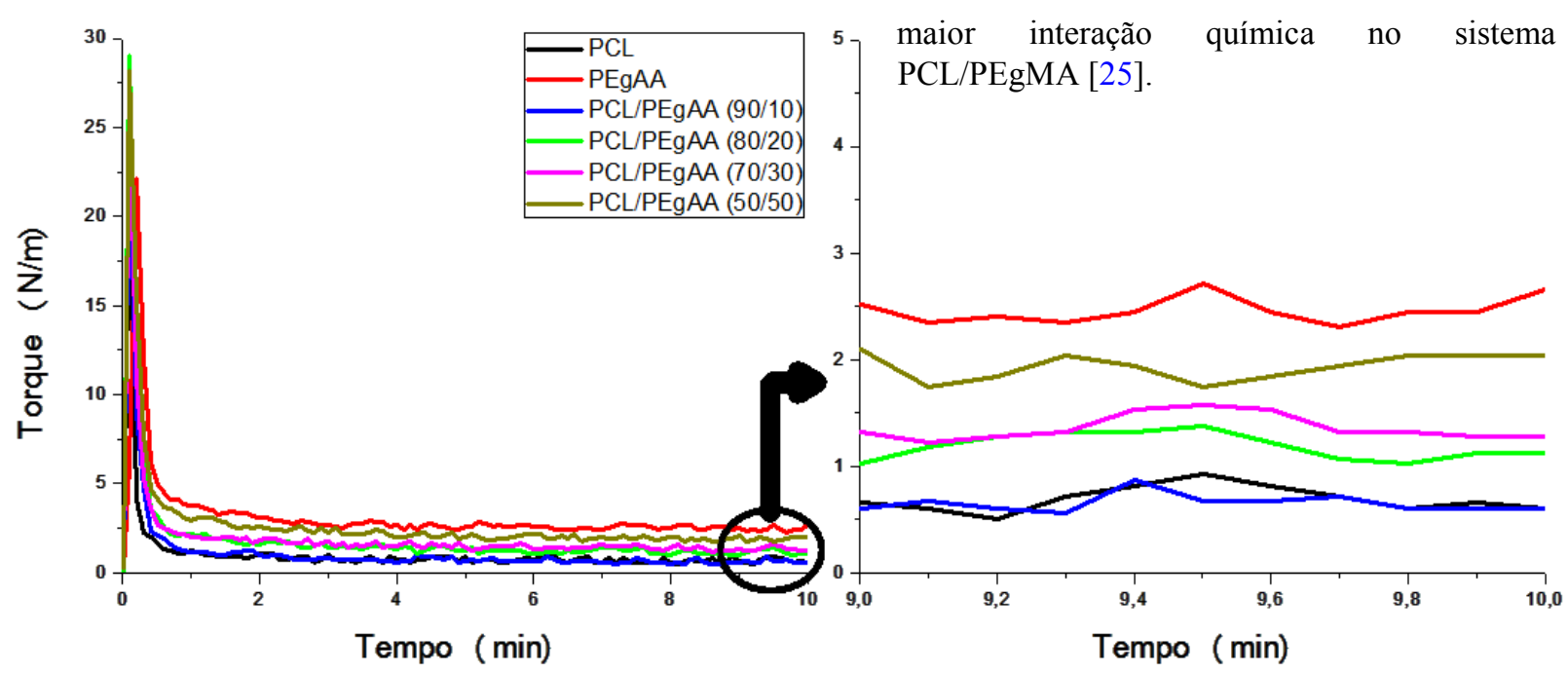

Figura 6: Curvas de torque em função do tempo de processamento para o PCL e os sistemas PCL/PEgAA: 90/10, 80/20, $70 / 30$ e $50 / 50$ (medidas em peso).
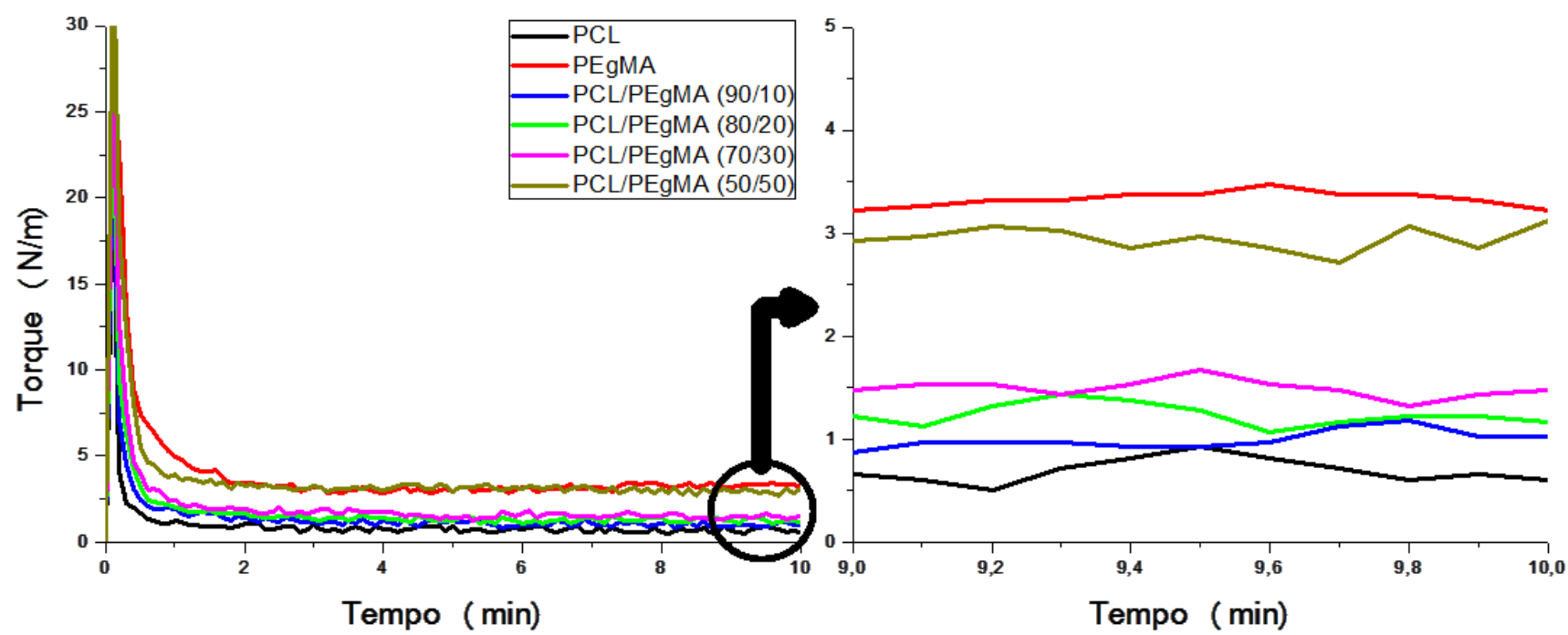

Figura 7: Curvas de torque em função do tempo de processamento para o PCL e os sistemas PCL/PEgMA: 90/10,80/20, $70 / 30$ e $50 / 50$ (medidas em peso).

Reações químicas entre os grupamentos do ácido acrílico e do anidrido maléico com o PCL são esperadas ocorrer, por estes serem polímeros polares (diferente do Bio-PE que é apolar) as quais podem também contribuir para o aumento da viscosidade/torque verificados nas Figuras 6 e 7.

Reação de poliesterificação entre o grupo terminal hidroxila do PCL e o grupo ácido carboxílico do PEgAA podem ter ocorrido durante o processamento, como ilustrado na Figura 8. De acordo com a literatura [26-29], grupos hidroxila podem reagir com grupos carboxila, via policondensação, para formar poliésteres, essa reação é capaz de gerar um grupo éster, onde a carbonila do ácido acrílico passa a compor esse grupo. Estando presente, essa reação contribui para aumento do peso molecular e então do torque dos sistemas $\mathrm{P}$ er inn . .

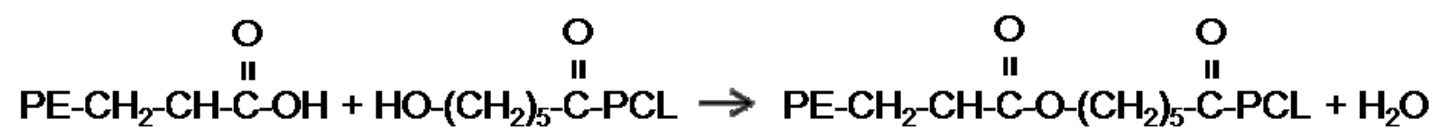

Figura 8: Reação de poliesterificação entre o grupo terminal hidroxila do PCL e o grupo ácido carboxílico do PEgAA. 
No caso do sistema PCL/PEgMA, o aumento no torque pode também ser resultantede reações de esterificação entre o anidrido maléico e os grupos hidroxilas terminais do PCL, conforme ilustrado na Figura 9.

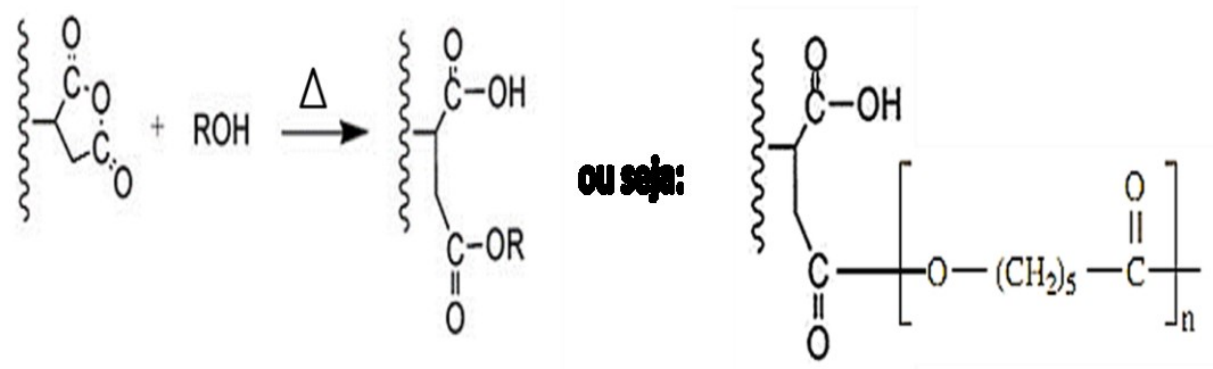

Figura 9: Reação de poliesterificação entre o grupo terminal hidroxila do PCL e o grupo ácido carboxílico do PEgMA.

Aumento significativo do torque foi observado para o sistema PCL/PEgMA (50/50), possivelmente devido a uma maior concentração de anidrido maleico no sistema disponível para reagir com as hidroxilas do PCL. Reações entre os grupos MA e poliéster foi previamente reportada por Chellavier et al. [30] e Souza [31].

Na Figura 10 são ilustradas as curvas da temperatura versus tempo de processamento para o PCL com os teores de 10, 20, 30 e 50\% de PEgAA e PEgMA. O mesmo padrão de temperatura é observado, ou seja, diminuição da temperatura durante a fase de alimentação e formação do plateau térmico, desta forma pode-se assumir que variações de viscosidade estão relacionadas a interações e/ou reações químicas e influência dos IF do PCL e dos polímeros enxertados.
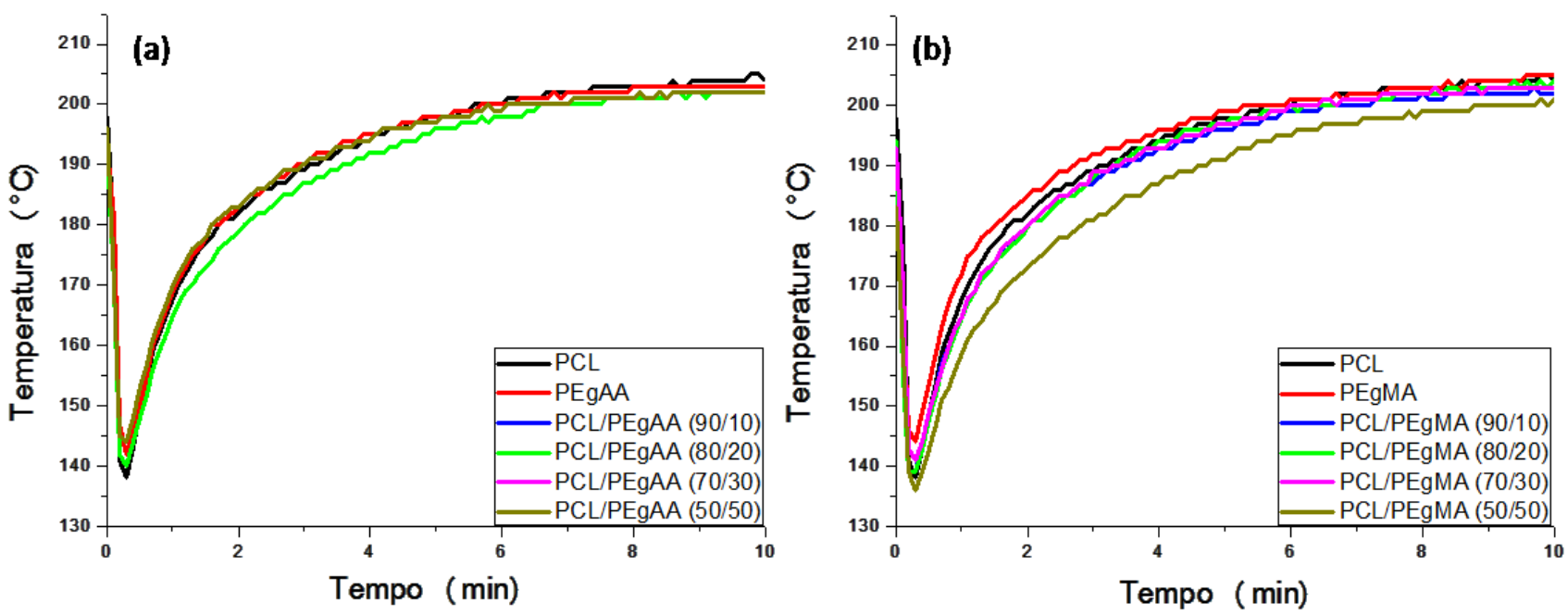

Figura 10: Curvas de temperatura em função do tempo de processamento: (a) PCL/PEgAA e (b) PCL/PEgMA.

A fim de se obter informações sobre possíveis reações entre os grupos funcionais dos polímeros BioPE e PCL com os compatibilizantes PEgMA e PEgAA, foi empregada a técnica de espectroscopia na região do infravermelho com transformada de Fourier (FTIR), a Figura 11 apresenta os espectros de FTIR dos sistemas poliméricos investigados neste trabalho.

Verifica-se para o Bio-PE bandas de absorbância localizadas em 2903 e 2838,1470 e $715 \mathrm{~cm}^{-1}$ associadas ao grupamento C-H [32]. Para o PEgAA são observadas bandas em 2914, 2850, 1468 e $726 \mathrm{~cm}^{-1}$ relacionadas com o estiramento do grupo $\mathrm{C}-\mathrm{H}$, e bandas em 2350 e $1713 \mathrm{~cm}^{-1}$, referente a frequência de estiramento de alcinos e ao estiramento da carbonila $(\mathrm{C}=\mathrm{O})$ do grupo ácido carboxílico proveniente da fração de ácido acrílico do polímero funcionalizado [28]. O PEgMA possui de forma análoga ao PEgAA bandas de absorção na faixa de 2915, 2837, 2351,1468 e $726 \mathrm{~cm}^{-1}$ devido ao estiramento do grupo C-H, e uma banda a $1724 \mathrm{~cm}^{-1}$, referente ao grupo anidrido maléico [32, 33]. 
Nos espectros das blendas pode-se observar as bandas referentes ao Bio-PE e aos polímeros enxertados. No caso do espectro das blendas Bio-PE/PEgAA (90/10 e 80/20) e Bio-PE/PEgMA (90/10, 80/20, 70/30 e 50/50) nota-se o desaparecimento do estiramento da carbonila do grupo ácido carboxílico e anidrido maleico, respectivamente. Além disso, os espectros do Bio-PE/PEgMA (80/20 e 50/50) apresentaram o surgimento de uma nova banda de absorção em $625 \mathrm{~cm}^{-1}$ referente ao grupo $\mathrm{C}=\mathrm{O}$ (Figura 11 (b)). De forma geral, os espectros das blendas Bio-PE/PEgAA e Bio-PE/PEgMA apresentaram indícios de uma possível solubilização entre o biopolímero e os compatibilizantes, corroborando com os resultados de reologia de torque apresentados nas Figuras 3 e 4.

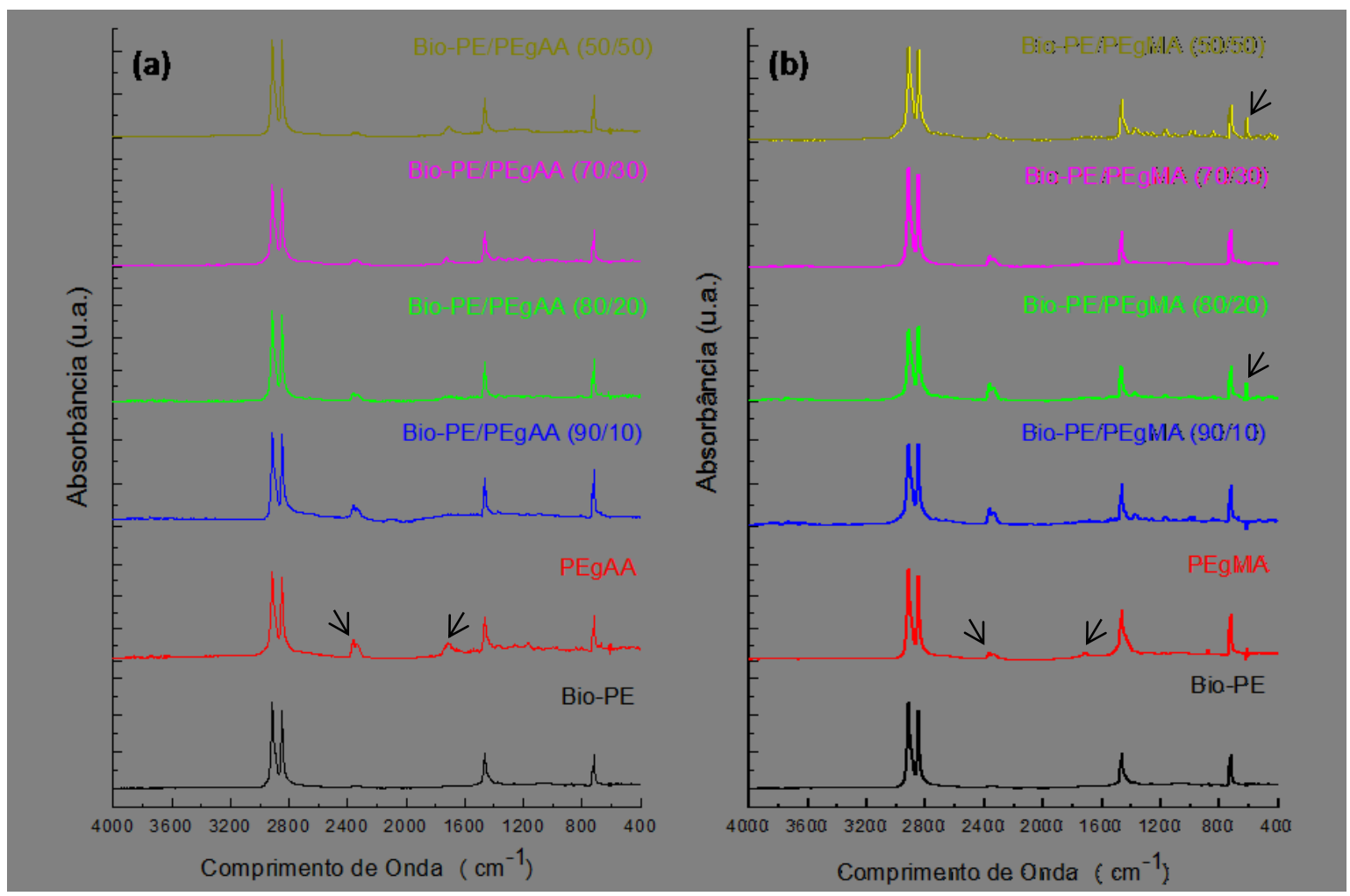

Figura 11: Detalhes dos espectros de FTIR: (a) Bio-PE/PEgAA e (b) Bio-PE/PEgMA.

No espectro de FTIR do PCL mostrado na Figura 12 observam-se bandas de absorção a 2952 e 2863 $\mathrm{cm}^{-1}$ atribuídos ao estiramento do grupamento $\mathrm{C}-\mathrm{H}$, a banda em $1724 \mathrm{~cm}^{-1}$ refere-se ao estiramento da carbonila $(\mathrm{C}=\mathrm{O})$ do grupo éster do PCL e bandas entre 1238 e $1162 \mathrm{~cm}^{-1}$ que são atribuídas ao estiramento da ligação do C-O-C [34, 35].

Na Figura 12 (a) e (b) tem-se os espectros de FTIR das blendas PCL/PEgAA e PCL/PEgMA. É possível observar a partir da Figura 12 (a) que a adição de diferentes teores de PEgAA não promoveu o surgimento de novas bandas de absorção, mas a adição desse polímero promoveu o desaparecimento das bandas referentes à frequência de estiramento de alcinos e ao desaparecimento ou sobreposição do estiramento da carbonila do grupo ácido carboxílico. Para a Figura 12 (b), observou-se um comportamento semelhante ao apresentado pela Figura 12 (a). De forma geral, os espectros das blendas PCL/PEgAA e PCL/PEgMA indicaram que pode ter havido reações entre o grupo terminal do PCL e o grupo ácido carboxílico (PEgAA) e do anidrido maléico (PEgMA), conforme apresentado nas Figuras 8 e 9 [29, 31, 36-39]. Os resultados apresentados na Figura 12 corroboram com as curvas de reometria mostradas nas Figuras 6 e 7. Em relação às blendas, observam-se também nos espectros de FTIR as principais bandas de absorção do PCL, além das bandas de absorção relacionadas ao grupo metileno do PEgAA e do PEgMA [28, 35]. 

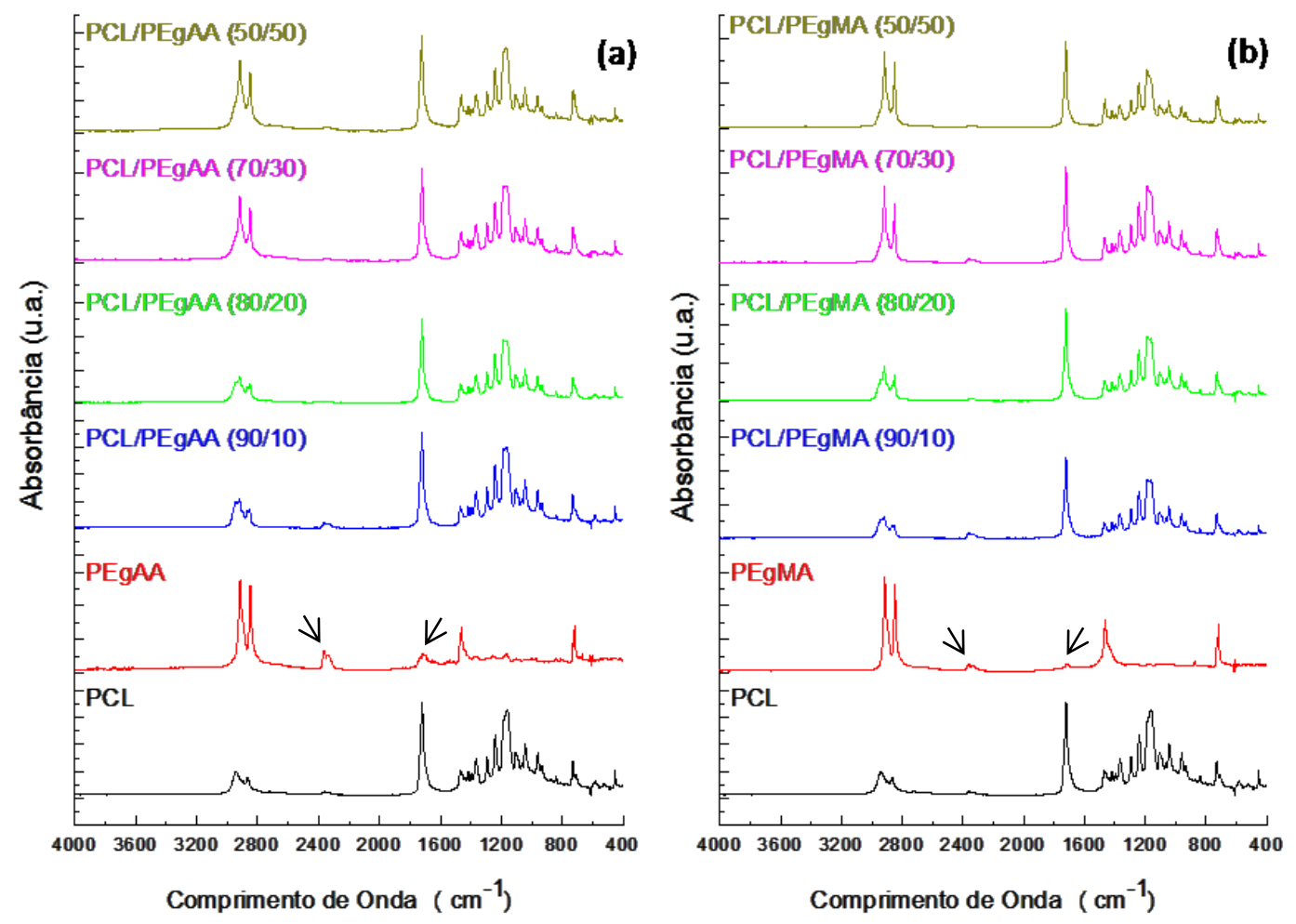

Figura 12: Detalhes dos espectros de FTIR: (a) PCL/PEgAA e (b) PCL/PEgMA.

\section{CONCLUSÕES}

O comportamento reológico dos sistemas poliméricos Bio-PE/PEgAA, Bio-PE/PEgMA, PCL/PEgAA e $\mathrm{PCL} / \mathrm{PEgMA}$ foi investigado através de experimentos de reometria de torque. As curvas de torque indicaram que o PEgAA e o PEgMA foram reativos com o PCL em relação aos sistemas com Bio-PE, sugerindo possíveis interações e/ou reações químicas entre os grupos funcionais dos polímeros enxertados com os grupos terminais do PCL, resultados estes também evidenciados por espectros de FTIR. Nas curvas reológicas de temperatura versus tempo observou-se que tanto os polímeros puros, quanto as blendas poliméricas apresentam, após a etapa de alimentação um plateau constante de temperatura, evidenciando que variações nas curvas de torque se originam de diferenças no IF, interações e/ou reações químicas, e não resultantes de variações de temperatura.

\section{AGRADECIMENTOS}

Os autores agradecem à Addivant pelos polímeros enxertados, ao Labmat (Laboratório de Engenharia de Materiais/CCT/UFCG) pelos experimentos realizados, ao MCTI/CNPq, CAPES/PNPD e à CAPES, pelo apoio financeiro.

\section{BIBLIOGRAFIA}

[1] HEMAIS, C. A., ROSA, E. O. R., BARROS, H. M., “Observações sobre o Desenvolvimento Tecnológicoe os Ciclos da Indústria de Polímeros no Brasil”, Polímeros: Ciência e Tecnologia, v. 10, n. 3, pp. 149-154, 2000 .

[2] HEMAIS, C. A., "Polímeros e a Indústria Automobilística", Polímeros: Ciência e Tecnologia, v. 13, n. 2 , pp. 107-114, 2003.

[3] MERALDO, A., "Introduction to Bio-Based Polymers", In: Wagner Jr, J. R, Multilayer Flexible Packaging, 2 ed., chapter 4, USA, Elsevier, 2016.

[4] COUTINHO, F. M. B., MELlO, I. L., MARIA, L. C. S., "Polietileno: Principais Tipos, Propriedades e Aplicações", Polímeros: Ciência e Tecnologia, v. 13, n. 1, pp. 1-13, 2003. 
[5] BRITO, G. F., AGRAWAL, P., ARAÚJO. E. M., MÉLO, T. J. A., "Polylactide/Biopolyethylene Bioblends", Polímeros: Ciência e Tecnologia, v. 22, n. 5, pp. 427-429, 2012.

[6] BRASKEM PRODUTOS VERDES, http://www.braskem.com.br/site.aspx/produtos-verdes. Acessado em agosto de 2015.

[7] ROSA, D. S., CHUI, Q. S. H., FILHO, R. P., et al., "Avaliação da Biodegradação de Poli- $\beta$ (Hidroxibutirato), Poli- $\beta$-(Hidroxibutirato-co-valerato) e Poli- $\varepsilon$-(caprolactona) em Solo Compostado", Polímeros: Ciência e Tecnologia, v. 12, n. 4, pp. 311-317, 2002.

[8] SWIFT, G., "Requirements for biodegradable water-soluble polymers", Polymer Degradation and Stability, v. 59, pp. 19-24, 1998.

[9] FRANÇA, D. C., BEZERRA, E. B., MORAIS, D. D. S., et al., "Hydrolytic and Thermal Degradation of PCL and PCL/Bentonite Compounds". Materials Research, v. 19, n. 3, pp. 618-627, 2016.

[10] FREITAS JUNIOR, N. F., Estudo das Propriedades Termomecânicas e Morfológicas de Blendas Biodegradáveis de Poli ( $\varepsilon$-Caprolactona) (PCL) com Amido de Milho Natural e Modificado. Dissertação de M.Sc., UNICAMP, Campinas, SP, Brasil, 2004.

[11] TAVARES, V. A. C. D. B., Matrizes de Policaprolactona e Quitosano para aplicação em Engenharia de Tecidos. Dissertação de M.Sc., UNL, Campolide, LX, Portugal, 2011.

[12] UTRACKI, L.A., Polymer Blends Handbook, Vol. 1, Netherlands, Kluwer Academic Publishers, 2002.

[13] ENRIQUEZ, E., MOHANTY, A.K., MISRA, M., "Biobased polymer blends of poly(trimethylene terephthalate) and high density polyethylene”, Materials \& Design, v. 90, pp. 984-990, 2016.

[14] SILVA, B. L., Influência do Agente Compatibilizante PE-co-PEG nas Propriedades da Blenda PE/Epóxi. Dissertação de M.Sc.,UDESC, Joinville, SC, Brasil, 2014.

[15] FOWER, J. N., SAITO, T., GAO, R., et al., "Impact of Diblock Copolymers on Droplet Coalescence, Emulsification, and Aggregation in Immiscible Homopolymer Blends", Langmuir, v. 28, n. 5, pp. 2347-2356, 2012.

[16] AGRAWAL, P., RODRIGUES, A. W. B., ARAÚJO, E.M., MÉLO, T. J.A., "Blendas de PA6/PE: avaliação da reatividade de diferentescompatibilizantes com a PA6 por reometria de torque", Revista Eletrônica de Materiais e Processos, v.4.3, pp. 01-10, 2009.

[17] AgRAWAL, P., ARAÚJo, E. M., MELO, T. J. A., "Reometria de Torque, Propriedades Mecânicas e Morfologia de Blendas Compatibilizadas de PA6/PEAD”, Polímeros: Ciência e Tecnologia, v. 18, n. 2, pp.152-157, 2008.

[18] Al-MALAIKA, S., KONG, W., "Reactive processing of polymers: effect of in situ compatibilisation on characteristics of blends of polyethylene terephthalate and ethylene-propylene rubber", Polymer, v. 46, n. 1, pp. 209-228, 2005.

[19] BRANDRUP, J., IMMERGUT, E. H., GRUlKe, E. A., Polymer Handbook, Vol. 2, Fourth Edition, United States of America, Wiley Interscience, 1999.

[20] HANSEN, C. M., Hansen Solubility Parameters, Second Edition, Boca Raton, CRC Press, 2007.

[21] KHALAF, M. N., AL-MOWALI, A. H., ADAM G. A., "Rheological Studies of Modified Maleated Polyethylene/Medium Density Polyethylene Blends”, Malaysian Polymer Journal, v. 3, pp. 54-64, 2008.

[22] LIU Y., LI K., "Development and characterization of adhesives from soy protein for bonding wood", International Journal of Adhesion \& Adhesives, v. 27, n. 1, pp. 59-67, 2007.

[23] MAURANO, C. H. F., GALlAND, G. B., MAULER, R.S., "Influência da Estrutura de Diferentes Copolímeros de Etileno e $\alpha$-Olefinas na Funcionalização com Anidrido Maleico", Polímeros Ciência e Tecnologia, v. 8, n. 3, pp. 79-88, 1998.

[24] SHIN, B. Y., HAN, D. H., “Compatibilization of immiscible poly(lacticacid)/poly( $\varepsilon$-caprolactone) blend through electron-beam irradiation with the addition of a compatibilizing agent", Radiation Physics and Chemistry, v. 83, pp. 98-104, 2013.

[25] HUANG, J-C., LIN, K-T., DEANIN, R.D., "Three-dimensional solubility parameters of poly( $\varepsilon$ caprolactone)", Journal of Applied Polymer, v.100, n. 3, pp. 2002-2009, 2006.

[26] TAKASU, A., OISHI, Y., LIO, Y., et al., "Synthesis of aliphatic polyesters by direct polyesterification of dicarboxylic acids with diols under mild conditions catalyzed by reusable rare-earth triflate", Macromolecules, v. 36, n. 6, pp. 1772-1774, 2003.

[27] VELMATHI, S., NAGAHATA, R., SUGIYAMA, J-I., et al., “A Rapid Eco-Friendly Synthesis of Poly 
(butylene succinate) by a Direct Polyesterification under Microwave Irradiation”, Macromolecular Rapid Communications, v. 26, n. 14, pp. 1163-1167, 2005.

[28] ARAÚJO, J. P., AGRAWAL, P. A., MÉLO, T. J. A., "Blendas PLA/PEgAA: Avaliação da reatividade entre os polímeros e da concentração de PEgAA nas propriedades e na morfologia", Revista Eletrônica de Materiais e Processos, v. 10, n. 3, pp. 118-127, 2015.

[29] SAINT-LOUP, R., ROBIN, J. J., BOUTEVIN, B., "Synthesis of Poly (ethylene terephthalate)-blockPoly (tetramethylene oxide) Copolymer by Direct Polyesterification of Reactive Oligomers", Macromolecular Chemistry and Physics, v. 204, n. 7, pp. 970- 982, 2003.

[30] CHEVALLIER, C., BECQUART, F., MAJESTE, J-C., TAHA, M., "Solvent-free preparation, characterization, and properties of SEBS-g-polycarbonate copolymers", Designed Monomers and Polymers, v. 6, n. 16, pp. 564-577, 2013.

[31] SOUZA, D. D., Desenvolvimento de Blendas de Poliestireno/Poli(E-caprolactona). Tese de D.Sc., UFCG, Campina Grande, PB, Brasil, 2016.

[32] KIM, Y. C., LEE, S. J., KIM, J. C., CHO, H., "Effect of Maleated Polyethylene on the Rheological Propertiesof LLDPE/Clay Nanocomposites", Polymer Journal, v. 37, n. 3, pp. 206-213, 2005.

[33] LUNA, C. B. B., SILVA, D. F., ARAÚJO, E. M., "Estudo do Comportamento de Blendas de Poliamida 6/Resíduo de Borracha da Indústria de Calçados", Revista Univap, v. 20, n. 36, pp. 98-110, 2014.

[34] ELZEIN, T., NASSER-EDDINE, M., DELAITE, C., BISTAC, S., DUMAS, P., "FTIR study of polycaprolactone chain organization at interfaces", Journal of Colloid and Interface Science, v. 273, pp. 381-387, 2004.

[35] CIARDELli, G., CHIONO, V., VOZZI, G., et al., "Blends of Poly-(e-caprolactone) and Polysaccharides in Tissue Engineering Applications", Biomacromolecules, v. 6, pp. 1961-1976, 2005.

[36] WU, C-H., "Physical properties and biodegradability of maleated-polycaprolactone/starch composite", Polymer Degradation and Stability, v. 80, pp. 127-134, 2003.

[37] LIU, N. C., BAKER, W. E., "Reactive Polymers for Blend Compatibilization", Advances in Polymer Technology, v. 11, n. 4, pp. 249-262, 1992.

[38] PORTER, R. S., WANG, L-H., "Compatibility and transesterification in binary polymer blends", Poly$m e r$, v. 33, n. 10, pp. 2019-2030, 1992.

[39] HUANG, C-Y., ROAN, M-L., KUO, M-C., LU, W-L., "Effect of compatibiliser on the biodegradation and mechanical properties of high-content starch/low-density polyethylene blends", Polymer Degradation and Stability, v. 90, pp. 95-105, 2005. 